TRANSACTIONS OF THE

AMERICAN MATHEMATICAL SOCIETY

Volume 352, Number 7, Pages 2993-3013

S 0002-9947(00)02518-6

Article electronically published on March 29, 2000

\title{
AN ELECTROMAGNETIC INVERSE PROBLEM IN CHIRAL MEDIA
}

\author{
STEPHEN R. MCDOWALL
}

\begin{abstract}
We consider the inverse boundary value problem for Maxwell's equations that takes into account the chirality of a body in $\mathbb{R}^{3}$. More precisely, we show that knowledge of a boundary map for the electromagnetic fields determines the electromagnetic parameters, namely the conductivity, electric permittivity, magnetic permeability and chirality, in the interior. We rewrite Maxwell's equations as a first order perturbation of the Laplacian and construct exponentially growing solutions, and obtain the result in the spirit of complex geometrical optics.
\end{abstract}

\section{INTRODUCTION}

In 12, Sylvester and Uhlmann proved that the conductivity of a body can be uniquely identified from information obtained only from the boundary. If a time dependence is introduced to the electromagnetic fields, the equations governing these fields change from a single second order elliptic partial differential equation to the full Maxwell's equations. In [11 Somersalo et al. presented a boundary map for time-harmonic fields at a fixed frequency, and raised the question of whether the parameters describing the electromagnetic properties of the body could be determined from knowledge of this boundary map. They showed that these parameters could be recovered approximately provided they differed only slightly from known constants. In [7] this assumption was dropped, and it was shown that the parameters are recoverable provided they are known in a small neighborhood of the boundary of the body.

In all these treatments, the constituent equations, which describe the dependence of the electric displacement and the magnetic induction on the electric and magnetic fields, do not take into account the chirality of the body. Instead, they depend only on the conductivity, electric permittivity and magnetic permeability of the body. Chirality is an asymmetry in the molecular structure; a molecule is chiral if it cannot be superimposed onto its mirror image. Presence of chirality results in the rotation of electromagnetic fields and is observable, particularly in the microwave range. Such experimental observations are used in physical chemistry to characterize molecular structures. For a detailed treatment of chirality and time-harmonic electromagnetic fields, see [2].

Received by the editors June 9, 1997.

1991 Mathematics Subject Classification. Primary 35R30, 35Q60; Secondary 35S15.

Key words and phrases. Inverse boundary value problems, Maxwell's equations, chirality, interior determination.

The author was partially supported by NSF Grant DMS-9705792. 
In this work we treat the case of a chiral body, and so the constituent equations depend on a fourth parameter $\beta$ which describes this chirality. In [8] Ola and Somersalo simplified the proof of interior identifiability in [7] by constructing a second order system of differential equations, which has as its principal part the Laplacian, in such a way that solutions to this system yields solutions to Maxwell's equations. They were able to construct a system with no first order part, that is a Schrödinger equation, and then use the results of [12] to construct exponentially growing solutions. Here we follow this idea and show that in the chiral case we are able to construct a system with the Laplacian as its principal part which again yields solutions to Maxwell's equations, but which has a first order term. Nakamura and Uhlmann [5] have developed a technique to handle such first order perturbations of the Laplacian, and it is this technique we employ here to construct exponentially growing solutions. The ability to construct these solutions enables us to use complex geometrical optics to prove identifiability of three of the material parameters throughout the body assuming knowledge of the fourth; in particular, assuming that the magnetic permeability is known, the chirality is determined uniquely by the boundary information.

In section 2 we state the problem precisely, present the main theorem (theorem 2.2), and briefly outline the proof, which comprises the later sections. Section 3 sets up the second order system; in section 4 we construct the exponentially growing solutions. The proof of our result is brought together in section [5. Sections [6] and [7are appendices including some more technical proofs.

\section{Statement of the Result}

Let $\Omega$ be a bounded connected subset of $\mathbb{R}^{3}$ with connected complement and with smooth boundary $\partial \Omega$. We restrict our interest to time-harmonic electromagnetic fields on $\Omega$, at fixed frequency $\omega$, i.e. if $E$ and $H$ are the electric and magnetic fields respectively then

$$
E=e^{i \omega t} E(x), \quad H=e^{i \omega t} H(x) .
$$

For such time-harmonic fields, Maxwell's equations are

$$
\nabla \wedge E=i \omega B, \quad \nabla \wedge H=-i \omega D .
$$

Using the Born-Fedorov formulation for a chiral body, (see [2]), the magnetic induction $B$ and the electric displacement $D$ are related to $E$ and $H$ through the constituent equations

$$
B=\tilde{\mu}(H+\tilde{\beta} \nabla \wedge H), \quad D=\tilde{\varepsilon}(E+\tilde{\beta} \nabla \wedge E) .
$$

Here $\tilde{\varepsilon}=\sigma+(i / \omega) \gamma$, where $\sigma$ is the electric permittivity and $\gamma$ is the conductivity, and $\tilde{\mu}$ is the magnetic permeability of the body. The chirality of the body is described by $\tilde{\beta}$. The parameters $\sigma, \gamma, \tilde{\mu}$ and $\tilde{\beta}$ are real-valued, and we assume here that $\tilde{\varepsilon}, \tilde{\mu}$ and $\tilde{\beta}$ are smooth and are constant outside a compact set. We assume

$$
\sigma \geq \sigma_{0}>0, \quad \gamma \geq 0, \quad \tilde{\mu} \geq \tilde{\mu}_{0}>0
$$

for constants $\sigma_{0}$ and $\tilde{\mu}_{0}$. We shall be using an equivalent formulation but with

$$
\varepsilon=\frac{\tilde{\varepsilon}}{1-\omega^{2} \tilde{\varepsilon} \tilde{\mu} \tilde{\beta}^{2}}, \quad \mu=\frac{\tilde{\mu}}{1-\omega^{2} \tilde{\varepsilon} \tilde{\mu} \tilde{\beta}^{2}}, \quad \beta=\frac{-i \omega \tilde{\varepsilon} \tilde{\mu} \tilde{\beta}}{1-\omega^{2} \tilde{\varepsilon} \tilde{\mu} \tilde{\beta}^{2}} .
$$


We are assuming that $1-\omega^{2} \tilde{\varepsilon} \tilde{\mu} \tilde{\beta}^{2} \neq 0$; this means we assume that the electric and magnetic fields never become parallel. Given the bounds (2.2), there is $\omega_{0}>0$ such that this assumption is satisfied for $\omega \in\left(-\omega_{0}, \omega_{0}\right)$; if $\omega \in\left(0, \omega_{0}\right)$, then $\varepsilon$ and $\mu$ are bounded away from zero, like $\tilde{\varepsilon}$ and $\tilde{\mu}$. With this change of parameters, we have the constituent equations

$$
B=\mu H-\beta E, \quad D=\varepsilon E+\beta H .
$$

We are assuming further that there are no magnetic poles or electric sinks or sources in $\Omega$; that is to say, we assume the induction and displacement to be divergence free:

$$
\nabla \cdot B=\nabla \cdot(\mu H-\beta E)=0, \quad \nabla \cdot D=\nabla \cdot(\varepsilon E+\beta H)=0 .
$$

We remark that $1-\omega^{2} \tilde{\varepsilon} \tilde{\mu} \tilde{\beta}^{2} \neq 0$ is equivalent to $\varepsilon \mu+\beta^{2} \neq 0$.

If $F$ is a function space, we denote by $F^{k}$ the space of $k$-vectors whose components are in $F$, and by $F^{k \times k}$ the space of $k \times k$ matrices whose components are in $F$. We shall need the following function spaces: $H^{s}(\Omega)^{k}$ consists of $k$-dimensional vector fields whose components are in the usual $L^{2}$-based Sobolev space $H^{s}$. Let Div denote the surface divergence on the boundary of $\Omega$, let $\nu(x)$ be the outward unit normal vector at $x \in \partial \Omega$, and define the following space of tangential fields:

$$
T H_{\text {Div }}^{\frac{1}{2}}(\partial \Omega)=\left\{F \in H^{\frac{1}{2}}(\partial \Omega)^{3} \mid \nu \cdot F=0, \text { and } \operatorname{Div} F \in H^{\frac{1}{2}}(\partial \Omega)\right\} .
$$

Theorem 2.1. Let $F \in T H_{\text {Div }}^{\frac{1}{2}}(\partial \Omega)$. There is a discrete set $D$ containing no limit points in $\left(0, \omega_{0}\right)$ such that for all $\omega \in\left(0, \omega_{0}\right) \backslash D$ there exist unique $(E, H) \in$ $\mathcal{D}^{\prime}(\Omega)^{3} \times \mathcal{D}^{\prime}(\Omega)^{3}$ solving the following boundary value problem:

$$
\begin{aligned}
& \nabla \wedge E=i \omega(\mu H-\beta E), \\
& \nabla \wedge H=-i \omega(\varepsilon E+\beta H), \\
& \left.\nu \wedge E\right|_{\partial \Omega}=F .
\end{aligned}
$$

We leave the proof of this to an appendix. We may thus define the boundary admittance map $\Pi: T H_{\text {Div }}^{\frac{1}{2}}(\partial \Omega) \rightarrow T H_{\text {Div }}^{\frac{1}{2}}(\partial \Omega)$ as follows. Given $F \in T H_{\text {Div }}^{\frac{1}{2}}(\partial \Omega)$, let $(E, H)$ solve $(2.5)$ and define

$$
\Pi F=\Pi\left(\left.\nu \wedge E\right|_{\partial \Omega}\right)=\left.\nu \wedge H\right|_{\partial \Omega} .
$$

The problem considered herein can now be stated.

If it is assumed a priori that $\mu_{1}=\mu_{2}=\mu$ (not necessarily constant) in $\Omega$, then we have the following:

Theorem 2.2. Let $\left(\Omega ; \varepsilon_{1}, \mu, \beta_{1}\right)$ and $\left(\Omega ; \varepsilon_{2}, \mu, \beta_{2}\right)$ be two electromagnetic bodies with the same smooth boundary $\partial \Omega$. Suppose that $\Pi_{1}=\Pi_{2}$; that is, if $F \in$ $T H_{\text {Div }}^{\frac{1}{2}}(\partial \Omega)$ and $\left(E_{j}, H_{j}\right)$ solve 2.5) with parameters $\left(\varepsilon_{j}, \mu, \beta_{j}\right)$ for $j=1,2$, then

$$
\Pi_{1} F=\left.\nu \wedge H_{1}\right|_{\partial \Omega}=\left.\nu \wedge H_{2}\right|_{\partial \Omega}=\Pi_{2} F
$$

If $\varepsilon_{1}=\varepsilon_{2}$ and $\beta_{1}=\beta_{2}$ on $\partial \Omega$, and the same is true of all normal derivatives at $\partial \Omega$, then

$$
\left(\varepsilon_{1}, \mu, \beta_{1}\right)=\left(\varepsilon_{2}, \mu, \beta_{2}\right)
$$

throughout $\Omega$. 
Remarks. (1) We can, in fact, show that if any of the three parameters are known a priori to agree in the body, then equality of the boundary maps implies agreement of the other parameters throughout the body.

(2) It was shown in [4] that $\Pi$ determines the material parameters and their first normal derivatives at the boundary. It is expected that the technique of [4] would show that $\Pi$ also determines all the higher order derivatives at the boundary; however, the computations become unmanageable.

(3) The assumption that the parameters agree to all order at the boundary is necessary only in the construction of the intertwining operators (see section 4). These operators belong to the Shubin class, which requires smooth symbols. In [13] Tolmasky showed that such intertwining operators may be constructed for equations with non-smooth parameters. This technique should remove the necessity of the assumption at the boundary, and also should lower the regularity assumptions on the parameters throughout $\Omega$.

(4) In the case that $\beta_{1}=\beta_{2}=0$, the result of [7] follows without any assumption on $\varepsilon$ or $\mu$ at $\partial \Omega$. The reason for this is that exponentially growing solutions are constructed without the need for intertwining operators, and so the parameters may be extended outside $\Omega$ in a non-smooth way.

We shall not impose the condition that $\mu_{1}=\mu_{2}$ until necessary at the end of the proof. Under the assumption of the theorem we may extend the parameters smoothly to all of $\mathbb{R}^{3}$ so that $\varepsilon_{1}=\varepsilon_{2}, \mu_{1}=\mu_{2}$ and $\beta_{1}=\beta_{2}$, outside $\Omega$, and so that $\varepsilon_{j}=\varepsilon_{0}, \mu_{j}=\mu_{0}, \beta_{j}=0, j=1,2$, outside a compact set containing $\Omega$. Here, $\varepsilon_{0}$ and $\mu_{0}$ are constants. Fundamental to the proof of theorem $[2.2$ is the following identity.

Proposition 2.3. Let $\left(E_{j}, H_{j}\right)$ solve 2.1) for parameters $\left(\varepsilon_{j}, \mu_{j}, \beta_{j}\right), j=1,2$. If $\Pi_{1}=\Pi_{2}$, then

$$
\int_{\Omega}\left(\left(\beta_{1}-\beta_{2}\right)\left(H_{1} \cdot E_{2}+H_{2} \cdot E_{1}\right)+\left(\varepsilon_{1}-\varepsilon_{2}\right) E_{1} \cdot E_{2}+\left(\mu_{2}-\mu_{1}\right) H_{1} \cdot H_{2}\right)=0
$$

Proof. Integrating by parts, and using the definition of $\Pi$, we get

$$
\begin{aligned}
\int_{\Omega} i \omega\left(\varepsilon_{1} E_{1}+\beta_{1} H_{1}\right) \cdot E_{2} & =-\int_{\Omega} \nabla \wedge H_{1} \cdot E_{2} \\
& =-\int_{\partial \Omega} \nu \wedge H_{1} \cdot E_{2}-\int_{\Omega} H_{1} \cdot \nabla \wedge E_{2} \\
& =-\int_{\partial \Omega} \Pi_{1} E_{1} \cdot E_{2}-\int_{\Omega} H_{1} \cdot i \omega\left(\mu_{2} H_{2}-\beta_{2} E_{2}\right)
\end{aligned}
$$

and similarly

$$
\int_{\Omega} i \omega\left(\varepsilon_{2} E_{2}+\beta_{2} H_{2}\right) \cdot E_{1}=-\int_{\partial \Omega} \Pi_{2} E_{2} \cdot E_{1}-\int_{\Omega} H_{2} \cdot i \omega\left(\mu_{1} H_{1}-\beta_{1} E_{1}\right) .
$$

Thus

$$
\begin{aligned}
& i \omega \int_{\Omega}\left(\left(\beta_{1}-\beta_{2}\right)\left(H_{1} \cdot E_{2}+H_{2} \cdot E_{1}\right)+\left(\varepsilon_{1}-\varepsilon_{2}\right) E_{1} \cdot E_{2}+\left(\mu_{2}-\mu_{1}\right) H_{1} \cdot H_{2}\right) \\
& =\int_{\partial \Omega}\left(\Pi_{2} E_{2} \cdot E_{1}-\Pi_{1} E_{1} \cdot E_{2}\right) .
\end{aligned}
$$


The proposition follows if we show that

$$
\int_{\partial \Omega} \Pi_{2} E_{2} \cdot E_{1}=\int_{\partial \Omega} E_{2} \cdot \Pi_{2} E_{1} .
$$

Let $\left(E_{0}, H_{0}\right)$ be the solution to (2.5) with parameters $\left(\varepsilon_{2}, \mu_{2}, \beta_{2}\right)$ and with $F=$ $\left.\nu \wedge E_{1}\right|_{\partial \Omega}$. Then

$$
\begin{aligned}
\int_{\partial \Omega}\left(\Pi_{2} E_{2} \cdot E_{1}\right. & \left.-E_{2} \cdot \Pi_{2} E_{1}\right)=\int_{\partial \Omega}\left(\nu \wedge H_{2} \cdot E_{1}-E_{2} \cdot \nu \wedge H_{0}\right) \\
& =\int_{\partial \Omega}\left(-H_{2} \cdot \nu \wedge E_{0}-E_{2} \cdot \nu \wedge H_{0}\right) \\
& =\int_{\Omega}\left(\nabla \wedge H_{2} \cdot E_{0}-H_{2} \cdot \nabla \wedge E_{0}-E_{2} \cdot \nabla \wedge H_{0}+\nabla \wedge E_{2} \cdot H_{0}\right) \\
& =\int_{\Omega}\left(-i \omega\left(\varepsilon_{2} E_{2}+\beta_{2} H_{2}\right) \cdot E_{0}-H_{2} \cdot i \omega\left(\mu_{2} H_{0}-\beta_{2} E_{0}\right)\right. \\
& \left.\quad+E_{2} \cdot i \omega\left(\varepsilon_{2} E_{0}+\beta_{2} H_{0}\right)+i \omega\left(\mu_{2} H_{2}-\beta_{2} E_{2}\right) \cdot H_{0}\right) \\
& =0 .
\end{aligned}
$$

The remainder of the paper is devoted to constructing sufficiently many suitable solutions to Maxwell's equations to conclude from (2.6) the claim of theorem 2.2 We present now an outline of the proof.

The aim is to use complex geometrical optics in the manner of [12] and many subsequent papers; that is, we wish to construct exponentially growing solutions depending on a complex parameter $\rho$ and to examine the asymptotics as the size of $\rho$ gets large. Rather than construct solutions to (2.1) directly, we follow the idea of Ola and Somersalo in [8] and introduce a new $8 \times 8$ system

$$
(P(\nabla)+V)\left(P(\nabla)+V^{\prime}\right) Y=(\Delta+N+Q) Y=0,
$$

where $P(\nabla)$ and $N$ are first order differential operators, and $V, V^{\prime}$ and $Q$ are matrix multipliers. We shall do this in such a way that if $Y$ is a solution to this system, and

$$
X=\left(P(\nabla)+V^{\prime}\right) Y
$$

is such that the first and last components of $X$ are zero, then the vector fields $\left(\left(X_{2}, X_{3}, X_{4}\right)^{\prime},\left(X_{5}, X_{6}, X_{7}\right)^{\prime}\right)$ will solve Maxwell's equations.

We then construct exponentially growing solutions to $(\Delta+N+Q) Y_{\rho}=0$ of the form

$$
Y_{\rho}=e^{x \cdot \rho}\left(y_{0, \rho}+\psi_{\rho}\right)
$$

with $\rho \in \mathbb{C}^{3}$ satisfying $\rho \cdot \rho=\omega^{2} \varepsilon_{0} \mu_{0}$, with $y_{0, \rho}$ an 8 -vector which is constant in $x$ and chosen to depend on $\rho$ in a convenient way, and $\psi_{\rho}$ constructed so that $\psi_{\rho} \rightarrow 0$ in some sense as $|\rho| \rightarrow \infty$. In [8], where chirality was not taken into account $(\beta=0)$, the above system included no first order term $N$, and so the authors were able to use the methods of [12] to construct exponentially growing solutions to a Schrödinger equation. When $\beta \neq 0$, such a reduction does not seem possible, and so here we must construct solutions to a first order perturbation of the Laplacian. The techniques employed are those of [5], where Nakamura and Uhlmann constructed solutions to a system of a similar form arising from elasticity. 
The final ingredient is to set $X_{\rho}=\left(P(\nabla)+V^{\prime}\right) e^{x \cdot \rho}\left(y_{0, \rho}+\psi_{\rho}\right)$ and to show that we can choose $y_{0, \rho}$ in such a way that $X_{\rho}$ yields solutions to Maxwell's equations, and to use these solutions in (2.6) to prove the claim of theorem 2.2

\section{A Reformulation of Maxwell's Equations}

In this section we introduce a new system of differential equations, the solutions of which, under certain restrictions, yield solutions to Maxwell's equations. We first introduce the following $8 \times 8$ operator:

$$
P(\nabla)=\left[\begin{array}{cccc}
0 & \nabla \cdot & 0 & 0 \\
\nabla & 0 & \nabla \wedge & 0 \\
0 & -\nabla \wedge & 0 & \nabla \\
0 & 0 & \nabla \cdot & 0
\end{array}\right]
$$

The domain of $P(\nabla)$ is $\mathcal{D}^{\prime}\left(\mathbb{R}^{3}\right) \times \mathcal{D}^{\prime}\left(\mathbb{R}^{3}\right)^{3} \times \mathcal{D}^{\prime}\left(\mathbb{R}^{3}\right)^{3} \times \mathcal{D}^{\prime}\left(\mathbb{R}^{3}\right)$. We point out that

$$
P(\nabla) P(\nabla)=\Delta \text {. }
$$

Our aim is to find $8 \times 8$ matrices $V$ and $V^{\prime}$ and write

$$
(P(\nabla)+V)\left(P(\nabla)+V^{\prime}\right)=\Delta+N+Q
$$

with $N$ a first order differential operator, and $Q$ a zero order matrix multiplier. Then if $Y$ solves

$$
(\Delta+N+Q) Y=0
$$

and we put

$$
X=\left(P(\nabla)+V^{\prime}\right) Y
$$

we would like (3.1) to imply that in some sense $X$ solves Maxwell's equations. The advantage of this reformulation is that we are in the position of seeking solutions to (3.1), for which a method is known.

We introduce some notation: for $X \in \mathcal{D}^{\prime}\left(\mathbb{R}^{3}\right) \times \mathcal{D}^{\prime}\left(\mathbb{R}^{3}\right)^{3} \times \mathcal{D}^{\prime}\left(\mathbb{R}^{3}\right)^{3} \times \mathcal{D}^{\prime}\left(\mathbb{R}^{3}\right)$ we shall write

$$
X=(a, A, B, b)^{\prime} .
$$

In order to have $X$ a solution to Maxwell's equations, we will find $Y$ in such a way that $a=b=0$; for the moment assume that this is the case. We must choose $V$ so that (3.1) implies (2.1) and (2.4); in particular, the central 6 rows of (3.1) must imply (2.1) and the first and last rows must imply (2.4). Let

$$
V_{m}=\left[\begin{array}{ll}
V_{22} & V_{23} \\
V_{32} & V_{33}
\end{array}\right] \quad \text { and } \quad L=i \omega\left[\begin{array}{cc}
\varepsilon I_{3} & \beta I_{3} \\
-\beta I_{3} & \mu I_{3}
\end{array}\right],
$$

where $V_{j k}$ are the $3 \times 3$ blocks in the center of $V$ and $I_{3}$ is the $3 \times 3$ identity matrix. If in fact $(A, B)$ are taken to be $(E, H)$ (that is, we don't rescale the fields in any way), then (3.1) is equivalent to

$$
0=\left(\begin{array}{c}
\nabla \wedge H \\
-\nabla \wedge E
\end{array}\right)=-V_{m}\left(\begin{array}{c}
E \\
H
\end{array}\right)
$$

and so, taking $V_{m}=L$, we obtain (2.1). Now set

$$
M=\left[\begin{array}{cc}
-\beta I_{3} & \mu I_{3} \\
\varepsilon I_{3} & \beta I_{3}
\end{array}\right], \quad \nabla M \cdot=\left[\begin{array}{cc}
-\nabla \beta \cdot & \nabla \mu \cdot \\
\nabla \varepsilon \cdot & \nabla \beta \cdot
\end{array}\right], \quad V_{0}=\left[\begin{array}{cc}
\vec{v}_{12} & \vec{v}_{13} \\
\vec{v}_{42} & \vec{v}_{43}
\end{array}\right],
$$


where $\vec{v}_{12}$ is the 3 -vector $\left(v_{12}, v_{13}, v_{14}\right), \vec{v}_{13}=\left(v_{15}, v_{16}, v_{17}\right), \vec{v}_{42}=\left(v_{82}, v_{83}, v_{84}\right)$, and $v_{43}=\left(v_{85}, v_{86}, v_{87}\right)$ in $V$. Notice that under the condition that $\varepsilon \mu+\beta^{2} \neq 0$, $M$ is invertible. Conditions (2.4) are equivalent to

$$
M\left(\begin{array}{c}
\nabla \cdot E \\
\nabla \cdot H
\end{array}\right)+\nabla M \cdot\left(\begin{array}{c}
E \\
H
\end{array}\right)=0, \quad \text { or } \quad\left(\begin{array}{c}
\nabla \cdot E \\
\nabla \cdot H
\end{array}\right)=-M^{-1} \nabla M \cdot\left(\begin{array}{c}
E \\
H
\end{array}\right),
$$

and $(P(\nabla)+V) X=0$ implies

$$
\left(\begin{array}{c}
\nabla \cdot E \\
\nabla \cdot H
\end{array}\right)+V_{0}\left(\begin{array}{c}
E \\
H
\end{array}\right)=0
$$

so putting

$$
V_{0}=M^{-1} \nabla M \cdot=\frac{1}{\varepsilon \mu+\beta^{2}}\left[\begin{array}{cc}
(\mu \nabla \varepsilon+\beta \nabla \beta) \cdot & (\mu \nabla \beta-\beta \nabla \mu) \cdot \\
(\beta \nabla \varepsilon-\varepsilon \nabla \beta) \cdot & (\varepsilon \nabla \mu+\beta \nabla \beta) \cdot
\end{array}\right]
$$

we have (2.4). At this point, assuming the first and last components $a$ and $b$ of $X$ are zero, we have determined the central 6 columns of $V$; so (3.1) implies that the fields $(A, B)$ satisfy 2.1. We now remove the assumption that $a=b=0$ and choose the rest of $V$ and all of $V^{\prime}$ in such a way that the equation

$$
(P(\nabla)+V)\left(P(\nabla)+V^{\prime}\right)=\Delta+N+Q
$$

has as simple a first order term $N$ as possible. This term is determined by $P(\nabla) V^{\prime}+$ $V P(\nabla)$; analyzing this row by row and making choices to eliminate first order terms, we find that we may choose

$$
V=\left[\begin{array}{cccc}
i \omega \mu & \vec{v}_{12} & \vec{v}_{13} & i \omega \beta \\
0 & i \omega \varepsilon & i \omega \beta & 0 \\
0 & -i \omega \beta & i \omega \mu & 0 \\
-i \omega \beta & \vec{v}_{42} & \vec{v}_{43} & i \omega \varepsilon
\end{array}\right], \quad V^{\prime}=\left[\begin{array}{cccc}
-i \omega \varepsilon & 0 & 0 & -i \omega \beta \\
0 & -i \omega \mu & -i \omega \beta & 0 \\
0 & i \omega \beta & -i \omega \varepsilon & 0 \\
i \omega \beta & 0 & 0 & -i \omega \mu
\end{array}\right]
$$

and obtain the first order term

$$
N=\left[\begin{array}{cccc}
\vec{v}_{12} \cdot \nabla & -\vec{v}_{13} \cdot \nabla \wedge & \vec{v}_{12} \cdot \nabla \wedge & \vec{v}_{13} \cdot \nabla \\
0 & 0 & 0 & 0 \\
0 & 0 & 0 & 0 \\
\vec{v}_{42} \cdot \nabla & -\vec{v}_{43} \cdot \nabla \wedge & \vec{v}_{42} \cdot \nabla \wedge & \vec{v}_{43} \cdot \nabla
\end{array}\right] .
$$

We remark that $N$ has compact support since its components consist of derivatives of the parameters, which are constant outside of a compact set. The zero order term $Q$ can be calculated easily, but as it will not be needed here we shall not present it explicitly. We shall use the fact that $Q-\omega^{2} \varepsilon_{0} \mu_{0} I$ has compact support.

Remark. A natural question to ask is, by rescaling the fields $(E, H)$ can a system be found that has no first order term, in which case we would have a Schrödinger equation? Such a system was achieved in [8] for a non-chiral body by rescaling the fields. For a chiral body, however, the answer to this appears to be no; suppose that we write $(A, B)=R(E, H)$ for some invertible matrix $R$ of the form

$$
R=\left[\begin{array}{ll}
r_{11} I_{3} & r_{12} I_{3} \\
r_{21} I_{3} & r_{22} I_{3}
\end{array}\right]
$$

and we set

$$
\widetilde{V}_{m}=\left[\begin{array}{cc}
-V_{32} & -V_{33} \\
V_{22} & V_{23}
\end{array}\right] \text { and } \widetilde{L}=i \omega\left[\begin{array}{cc}
-\beta I_{3} & \mu I_{3} \\
-\varepsilon I_{3} & -\beta I_{3}
\end{array}\right] .
$$


Then we find that to satisfy (2.1) we must set

$$
\begin{aligned}
\widetilde{V}_{m} & =-\nabla R \wedge R^{-1}-R \widetilde{L} R^{-1}, \\
V_{0} & =-\nabla R \cdot R^{-1}+R M^{-1} \nabla M \cdot R^{-1}
\end{aligned}
$$

(the notation should be interpreted in the way that makes sense), and this results in a first order term whose non-zero components are given by the components of

$$
-2 \nabla R \cdot R^{-1}+R M^{-1} \nabla M \cdot R^{-1}
$$

we conjecture that there is no choice of matrix $R$ which makes this zero. In [8] the rescaling matrix is

$$
R=\left[\begin{array}{cc}
\varepsilon^{\frac{1}{2}} & 0 \\
0 & \mu^{\frac{1}{2}}
\end{array}\right]
$$

and an easy calculation shows that the first order term vanishes when $\beta=0$. An interesting observation is that no matter what choice of $R$ is made, the system obtained by following this construction always leads to solutions to Maxwell's equations. The proof of this is more involved than what is presented here, but the same program carries through.

\section{Construction of Solutions - Intertwining Operators}

Recall that we wish to construct solutions to $(\Delta+N+Q) Y=0$ with $Y$ of the form $Y=e^{x \cdot \rho}\left(y_{0, \rho}+\psi_{\rho}\right)$. For $\rho \in \mathbb{C}^{3}$ with $\rho \cdot \rho=\omega^{2} \varepsilon_{0} \mu_{0}$ we define the operators

$$
\Delta_{\rho}=e^{-x \cdot \rho} \Delta\left(e^{x \cdot \rho} \cdot\right) \quad \text { and } \quad N_{\rho}^{+}=e^{-x \cdot \rho}\left(N+Q-\omega^{2} \varepsilon_{0} \mu_{0}\right)\left(e^{x \cdot \rho} \cdot\right),
$$

and so we wish to solve

$$
\left(\Delta_{\rho}+N_{\rho}^{+}\right)\left(y_{0, \rho}+\psi_{\rho}\right)=0 .
$$

We specify $\psi_{\rho}$ later by prescribing its asymptotic behavior. Generally speaking, our approach is to construct pseudodifferential operators $A_{\rho}, B_{\rho}$ and $C_{\rho}$ of order zero and depending on the parameter $\rho$ so that

$$
\left(\Delta_{\rho}+N_{\rho}^{+}\right) A_{\rho}\left(y_{0, \rho}+\psi_{\rho}\right)=B_{\rho}\left(\Delta_{\rho}+C_{\rho}\right)\left(y_{0, \rho}+\psi_{\rho}\right) .
$$

For sufficiently large $\rho, A_{\rho}$ is invertible, and we shall always take our operators to be properly supported, so that there is no problem defining compositions. This reduction to a zero order perturbation of the Laplacian enables us to use the extensive literature on constructing exponentially growing solutions. Such solutions have been used extensively in identifiability results, starting with the conductivity result of 12 .

We introduce the class these "intertwining operators" belong to. Let $Z=$ $\left\{\rho \in \mathbb{C}^{3}|| \rho \mid \geq 1, \rho \cdot \rho=\omega^{2} \varepsilon_{0} \mu_{0}\right\}$, and denote by $L^{0}\left(\mathbb{R}^{3}, Z\right)$ the Shubin class of order zero (see [10], section 9). We refer the reader to [5] for a discussion of the Shubin class of operators, and repeat some important properties here. Most importantly we define the symbol class of $L^{0}\left(\mathbb{R}^{3}, Z\right)$.

Definition 4.1. Let $\rho \in Z$; then $a_{\rho}(x, \xi) \in S^{0}\left(\mathbb{R}^{3}, Z\right)$ if and only if

1. $a_{\rho} \in C^{\infty}\left(\mathbb{R}^{3} \times \mathbb{R}^{3}\right)$ for each fixed $\rho \in Z$, and

2. for any multi-indices $\alpha, \delta$ and compact set $K \subset \mathbb{R}^{3}$, there exists a constant $C_{\alpha, \delta, K}>0$ such that 


$$
\sup _{x \in K}\left|\partial_{\xi}^{\alpha} \partial_{x}^{\delta} a_{\rho}(x, \xi)\right| \leq C_{\alpha, \delta, K}(1+|\xi|+|\rho|)^{-|\alpha|}
$$

for any $\xi \in \mathbb{R}^{3}, \rho \in Z$.

We say that $a_{\rho}$ is the full symbol of $A_{\rho}$ in the same way as for usual pseudodifferential operators. We say $A_{\rho} \in L^{0}\left(\mathbb{R}^{3}, Z\right)$ is properly supported if there exists a closed set $H \subset \mathbb{R}^{3} \times \mathbb{R}^{3}$ such that the support of the Schwartz kernel of $A_{\rho}$ is contained in $H$ for all $\rho \in Z$ and the projection of $H$ onto each factor $\mathbb{R}^{3}$ is proper. We note that if $A_{\rho} \in L^{0}\left(\mathbb{R}^{3}, Z\right)$ is properly supported, then we may expand the symbol $\widetilde{\sigma}\left(A_{\rho}\right)(x, \xi)$ of $A_{\rho}$ asymptotically as

$$
\left.\widetilde{\sigma}\left(A_{\rho}\right)(x, \xi) \sim \sum_{\alpha} \frac{1}{\alpha !} \partial_{\xi}^{\alpha} D_{y}^{\alpha} a_{\rho}(x, y, \xi)\right|_{y=x} .
$$

Proposition 4.2. Let $\varphi \in C_{c}^{\infty}\left(\mathbb{R}^{3}\right)$ be such that $\varphi$ is identically one on $\Omega$. Then there exist operators $A_{\rho}, B_{\rho}$ and $C_{\rho}$ in $L^{0}\left(\mathbb{R}^{3}, Z\right)^{8 \times 8}$ such that

$$
\left(\Delta_{\rho}+N_{\rho}^{+}\right) A_{\rho}=B_{\rho}\left(\Delta_{\rho}+\varphi C_{\rho} \varphi\right)
$$

We leave the proof of this to a later section. Let

$$
L_{\delta}^{2}=\left\{f \in L_{l o c}^{2}\left(\mathbb{R}^{3}\right):\|f\|_{\delta}^{2}=\int\left(1+|x|^{2}\right)^{\delta}|f(x)|^{2} d x<\infty\right\},
$$

and for $s \in \mathbb{R}$ let $H_{\delta}^{s}$ be the associated weighted Sobolev space. Assuming (4.2), we have the following proposition:

Proposition 4.3. Let $-1<\delta<0$, and let $y_{0, \rho}$ be an 8-vector constant in $x$ and bounded in $\rho$. Then for sufficiently large $|\rho|$ there exist $a \psi_{\rho} \in H_{\delta}^{2}\left(\mathbb{R}^{3}\right)^{8}$ and a constant $C$, depending only on $\delta, \varphi$ and $C_{\rho}$, such that

$$
\left(\Delta_{\rho}+\varphi C_{\rho} \varphi\right)\left(y_{0, \rho}+\psi_{\rho}\right)=0
$$

and

$$
\left\|\psi_{\rho}\right\|_{H_{\delta}^{2}} \leq \frac{C}{|\rho|} .
$$

Proof. We have $\Delta_{\rho} \psi_{\rho}=-\varphi C_{\rho} \varphi y_{0, \rho}-\varphi C_{\rho} \varphi \psi_{\rho}$; by [10], $C_{\rho}: H^{2}\left(\mathbb{R}^{3}\right)^{8} \rightarrow H^{2}\left(\mathbb{R}^{3}\right)^{8}$ continuously with operator norm independent of $\rho$, and since $\varphi$ is compactly supported, $\varphi C_{\rho} \varphi y_{0, \rho} \in H_{\delta+1}^{2}\left(\mathbb{R}^{3}\right)^{8}$. Let $r_{0}>0$; by [12], if $|\rho|>r_{0}>0$, we may solve

$$
\Delta_{\rho} \psi_{\rho}^{(0)}=-\varphi C_{\rho} \varphi y_{0, \rho}
$$

for $\psi_{\rho}^{(0)} \in H_{\delta}^{2}\left(\mathbb{R}^{3}\right)^{8}$, and, from the estimates for $\Delta_{\rho}^{-1}$ in $[12$,

$$
\left\|\psi_{\rho}^{(0)}\right\|_{H_{\delta}^{2}} \leq \frac{C\left(r_{0}, \delta\right)}{|\rho|}\left\|\varphi C_{\rho} \varphi y_{0, \rho}\right\|_{H_{\delta+1}^{2}} .
$$

In general, for any $j, \varphi C_{\rho} \varphi \psi_{\rho}^{(j-1)} \in H_{\delta+1}^{2}\left(\mathbb{R}^{3}\right)^{8}$, and so for $|\rho|>r_{0}$ we solve

$$
\Delta_{\rho} \psi_{\rho}^{(j)}=-\varphi C_{\rho} \varphi \psi_{\rho}^{(j-1)}
$$

with

$$
\left\|\psi_{\rho}^{(j)}\right\|_{H_{\delta}^{2}} \leq \frac{C\left(r_{0}, \delta\right)}{|\rho|}\left(\frac{C^{\prime}\left(\varphi, C_{\rho}\right)}{|\rho|}\right)^{j}\left\|\varphi C_{\rho} \varphi y_{0, \rho}\right\|_{H_{\delta+1}^{2}}
$$


choosing $|\rho|$ large enough and putting $\psi_{\rho}=\sum_{j=0}^{\infty} \psi_{\rho}^{(j)}$, we have $\psi_{\rho} \in H_{\delta}^{2}\left(\mathbb{R}^{3}\right)^{8}$ for sufficiently large $|\rho|$, and

$$
\left\|\psi_{\rho}\right\|_{H_{\delta}^{2}} \leq \frac{C}{|\rho|} .
$$

Furthermore, $\left(\Delta_{\rho}+\varphi C_{\rho} \varphi\right)\left(y_{0, \rho}+\psi_{\rho}\right)=0$.

Thus we have a means to construct solutions to (3.1). We have

$$
\left(\Delta_{\rho}+N_{\rho}^{+}\right) A_{\rho}\left(y_{0, \rho}+\psi_{\rho}\right)=0
$$

and introducing a cut-off to gain compact support, we put

$$
Y_{\rho}=e^{x \cdot \rho} \varphi A_{\rho}\left(y_{0, \rho}+\psi_{\rho}\right)
$$

then in $\Omega$ we have $(\Delta+N+Q) Y_{\rho}=0$. In order to construct solutions $Y_{\rho}$ so that $X_{\rho}=\left(P(\nabla)+V^{\prime}\right) Y_{\rho}$ are solutions to Maxwell's equations, we must ensure that the first and last components of $X_{\rho}$, namely $(a, b)$, are zero. We introduce the notation $P(\rho)$ to be the $8 \times 8$ matrix where $\rho$ replaces $\nabla$ in $P(\nabla)$.

Proposition 4.4. If $y_{0, \rho}$ is chosen so that the first and last components of $P(\rho) A_{\rho} y_{0, \rho}$ are zero, then the first and last components $(a, b)^{\prime}$ of $X_{\rho}=$ $\left(P(\nabla)+V^{\prime}\right) Y_{\rho}$ are zero.

Proof. Since $(P(\nabla)+V) X_{\rho}=0$, computing

$$
\left(\begin{array}{cccc}
-i \omega \varepsilon & \nabla \cdot & 0 & -i \omega \beta \\
i \omega \beta & 0 & \nabla \cdot & -i \omega \mu
\end{array}\right)(P(\nabla)+V) X_{\rho}=0,
$$

we obtain

$$
\Delta\left(\begin{array}{l}
a \\
b
\end{array}\right)+\omega^{2}\left(\begin{array}{cc}
\varepsilon \mu-\beta^{2} & 2 \varepsilon \beta \\
-2 \mu \beta & \varepsilon \mu-\beta^{2}
\end{array}\right)\left(\begin{array}{l}
a \\
b
\end{array}\right)=0
$$

Now

$$
\begin{aligned}
X_{\rho}= & \left(P(\nabla)+V^{\prime}\right) Y_{\rho}=\left(P(\nabla)+V^{\prime}\right) e^{x \cdot \rho} \varphi A_{\rho}\left(y_{0, \rho}+\psi_{\rho}\right) \\
= & e^{x \cdot \rho}\left\{P(\rho) \varphi A_{\rho} y_{0, \rho}+P(\rho) \varphi A_{\rho} \psi_{\rho}+P(\nabla) \varphi A_{\rho}\left(y_{0, \rho}+\psi_{\rho}\right)\right. \\
& \left.\quad+V^{\prime} \varphi A_{\rho}\left(y_{0, \rho}+\psi_{\rho}\right)\right\} \\
= & e^{x \cdot \rho}\left\{P(\rho) \varphi A_{\rho} y_{0, \rho}+X_{s}\right\}, \quad \text { say. }
\end{aligned}
$$

Writing

$$
\left(\begin{array}{c}
a \\
b
\end{array}\right)=e^{x \cdot \rho}\left\{\left(\begin{array}{c}
a_{0} \\
b_{0}
\end{array}\right)+\left(\begin{array}{c}
a_{s} \\
b_{s}
\end{array}\right)\right\},
$$

we have

$$
\left(\Delta+\omega^{2} \varepsilon_{0} \mu_{0}+q\right)\left(\begin{array}{l}
a \\
b
\end{array}\right)=0
$$

with

$$
q=\omega^{2}\left(\begin{array}{cc}
\varepsilon \mu-\beta^{2} & 2 \varepsilon \beta \\
-2 \mu \beta & \varepsilon \mu-\beta^{2}
\end{array}\right)-\omega^{2} \varepsilon_{0} \mu_{0}
$$

having compact support. Thus we have

$$
\Delta_{\rho}\left(\begin{array}{l}
a_{s} \\
b_{s}
\end{array}\right)+q\left(\begin{array}{l}
a_{s} \\
b_{s}
\end{array}\right)=-q\left(\begin{array}{l}
a_{0} \\
b_{0}
\end{array}\right) .
$$


Now $X_{s} \in L_{l o c}^{2}\left(\mathbb{R}^{3}\right)^{8}$ and has compact support, so in particular $\left(a_{s}, b_{s}\right)^{\prime} \in L_{\delta}^{2}\left(\mathbb{R}^{3}\right)^{2}$. By [12], $\left(a_{s}, b_{s}\right)^{\prime}$ is the unique solution in $L_{\delta}^{2}\left(\mathbb{R}^{3}\right)^{2}$, and since $\left(a_{0}, b_{0}\right)^{\prime}=(0,0)^{\prime}$, the proposition follows.

We will show later, in the proof of proposition 4.2, that the symbol $a_{\rho}(x, \xi)$ of $A_{\rho}$ is of the form

$$
\left[\begin{array}{ccccc}
a_{11} & a_{12} & \cdots & a_{17} & a_{18} \\
0 & \ddots & & & 0 \\
\vdots & & I_{6} & & \vdots \\
0 & & & \ddots & 0 \\
a_{81} & a_{82} & \cdots & a_{87} & a_{88}
\end{array}\right]
$$

where $I_{6}$ is the $6 \times 6$ identity matrix, and that $a_{\rho}(x, \xi)$ is homogeneous of degree zero in $\xi$ and $\rho$. Thus

$$
P(\rho) \varphi A_{\rho} y_{0, \rho}=\varphi\left[\begin{array}{c}
\rho \cdot\left(y_{2}, y_{3}, y_{4}\right) \\
\left(\vec{a}_{1} \cdot y_{0, \rho}\right) \rho+\rho \wedge\left(y_{5}, y_{6}, y_{7}\right) \\
\left(\vec{a}_{8} \cdot y_{0, \rho}\right) \rho-\rho \wedge\left(y_{2}, y_{3}, y_{4}\right) \\
\rho \cdot\left(y_{5}, y_{6}, y_{7}\right)
\end{array}\right],
$$

where $y_{j}$ are the components of $y_{0, \rho}$ and $\vec{a}_{1}$. and $\vec{a}_{8}$. are the first and last rows of $a_{\rho}$. To satisfy the conditions of proposition 4.4 we must therefore choose $y_{0, \rho}$ so that $\rho \cdot\left(y_{2}, y_{3}, y_{4}\right)=\rho \cdot\left(y_{5}, y_{6}, y_{7}\right)=0$.

\section{Proof of Theorem 2.2}

We first investigate the asymptotics in $\rho$ of $A_{\rho}$.

Proposition 5.1. If $f \in L^{2}(\Omega)^{8}$, then, for all $x \in \Omega$,

$$
A_{\rho} f(x)=a_{\rho}(x, 0) f(x)+R_{\rho} f(x)
$$

modulo smoothing, and

$$
\left\|R_{\rho} f\right\|_{L^{2}(\Omega)} \leq \frac{C}{1+|\rho|}\|f\|_{L^{2}(\Omega)}
$$

for a constant $C>0$ independent of $\rho$ and $f$. Recall that $a_{\rho}(x, \xi)$ is the symbol of $A_{\rho}$.

Proof. Let $\chi \in C_{c}^{\infty}\left(\mathbb{R}^{3}\right)$ be such that $\chi(x)=1$ on $\{|x| \leq 1\}, \chi(x)=0$ on $\{|x| \geq 2\}$, and let $\sigma(y) \in C_{c}^{\infty}\left(\mathbb{R}^{3}\right)$ be such that

$$
\sigma(y)=1 \text { on }\{y \mid \exists x \in \Omega \text { with } \chi(x-y) \neq 0\} .
$$

For $x \in \Omega$,

$$
\begin{aligned}
A_{\rho} f(x)= & \int e^{i(x-y) \cdot \xi} \chi(x-y) a_{\rho}(x, \xi) f(y) d y d \xi \\
& +\int e^{i(x-y) \cdot \xi}(1-\chi)(x-y) a_{\rho}(x, \xi) f(y) d y d \xi \\
= & \int e^{i(x-y) \cdot \xi} \chi(x-y) a_{\rho}(x, \xi)(\sigma f)(y) d y d \xi+g_{1}(x),
\end{aligned}
$$


where $g_{1} \in C^{\infty}\left(\mathbb{R}^{3}\right)^{8}$, since the second integral is smoothing. Here we have used $\sigma=1$, where $\chi(x-y) \neq 0$. Expanding $\chi$ in a Taylor series about $y=x$, we have, modulo smoothing,

$$
\begin{aligned}
A_{\rho} f(x) & =\int e^{i(x-y) \cdot \xi} a_{\rho}(x, \xi)(\sigma f)(y) d y d \xi \\
& =\int e^{i x \cdot \xi} a_{\rho}(x, \xi) \widehat{(\sigma f)}(\xi) d \xi
\end{aligned}
$$

where $\widehat{g}$ denotes the Fourier transform of $g$. We now expand $a_{\rho}(x, \xi)$ in a Taylor series about $\xi=0$ to obtain

$$
\begin{aligned}
A_{\rho} f(x)= & \left.\int e^{i x \cdot \xi} a_{\rho}(x, 0) \widehat{(\sigma f}\right)(\xi) d \xi \\
& +\int e^{i x \cdot \xi} \sum_{j=1}^{3} \xi_{j} \int_{0}^{1}\left(\partial_{\xi_{j}} a_{\rho}\right)(x, t \xi) d t \widehat{(\sigma f)}(\xi) d \xi \\
= & a_{\rho}(x, 0) f(x)+R_{\rho}^{(1)}(\sigma f)(x)
\end{aligned}
$$

modulo smoothing. Let $\tilde{\sigma}\left(R_{\rho}^{(1)}\right)$ denote the symbol of $R_{\rho}^{(1)}$; on the one hand, since $a_{\rho} \in S^{0}\left(\mathbb{R}^{3}, Z\right)^{8 \times 8}$, we have

$$
\left|\tilde{\sigma}\left(R_{\rho}^{(1)}\right)\right|=\left|\sum_{j=1}^{3} \xi_{j} \int_{0}^{1}\left(\partial_{\xi_{j}} a_{\rho}\right)(x, t \xi) d t\right| \leq \frac{C|\xi|}{1+|\rho|}
$$

for some constant $C>0$; but on the other hand, since $\tilde{\sigma}\left(R_{\rho}^{(1)}\right)=a_{\rho}(x, \xi)-a_{\rho}(x, 0)$, it is homogeneous of degree 0 in $\xi$ and $\rho$, and so

$$
\left|\tilde{\sigma}\left(R_{\rho}^{(1)}\right)\right| \leq \frac{C}{1+|\rho|} .
$$

Therefore,

$$
\left\|R_{\rho}^{(1)}(\sigma f)\right\|_{L^{2}(\Omega)} \leq \frac{C}{1+|\rho|}\|\sigma f\|_{L^{2}\left(\mathbb{R}^{3}\right)} \leq \frac{C}{1+|\rho|}\|f\|_{L^{2}(\Omega)} .
$$

We have denoted $R_{\rho}(f)=R_{\rho}^{(1)}(\sigma f)$.

We will need to know the asymptotics of derivatives of $X_{\rho}$, and so will use the following corollaries.

Corollary 5.2. If $f \in H^{2}(\Omega)^{8}$, then there exists a constant $C>0$, independent of $f$ and $\rho$, such that

$$
\begin{aligned}
P(\nabla) A_{\rho} f(x)= & \left(P(\nabla) a_{\rho}(x, 0)\right) f(x)+a_{\rho}(x, 0)(P(\nabla) f)(x) \\
& +R_{\rho}(P(\nabla) f)(x)+R_{\rho}^{\prime} f(x)
\end{aligned}
$$

modulo smoothing, and with

$$
\left\|R_{\rho}\right\|_{L^{2}(\Omega), L^{2}(\Omega)}+\left\|R_{\rho}^{\prime}\right\|_{L^{2}(\Omega), L^{2}(\Omega)} \leq \frac{C}{1+|\rho|} .
$$

Here $\|\cdot\|_{L^{2}(\Omega), L^{2}(\Omega)}$ denotes the operator norm. 
Corollary 5.3. If $X_{\rho}=\left(P(\nabla)+V^{\prime}\right) Y_{\rho}$ and $Y_{\rho}$ is as in 4.4), then in $\Omega$,

$$
\begin{aligned}
X_{\rho}=e^{x \cdot \rho}\left\{P(\rho) a_{\rho}(x, 0) y_{0, \rho}+P(\rho) R_{\rho} y_{0, \rho}+P(\rho) a_{\rho}(x, 0) \psi_{\rho}\right. \\
\\
\left.\quad+\left(P(\nabla) a_{\rho}(x, 0)\right) y_{0, \rho}+V^{\prime} a_{\rho}(x, 0) y_{0, \rho}+W_{\rho}\right\}
\end{aligned}
$$

and there is a constant $C>0$ such that

$$
\left\|W_{\rho}\right\|_{L^{2}(\Omega)} \leq \frac{C}{|\rho|} .
$$

Let $F_{1}$ and $F_{2}$ be the projections so that $F_{1} X=E=\left(X_{2}, X_{3}, X_{4}\right)$ and $F_{2} X=$ $H=\left(X_{5}, X_{6}, X_{7}\right)$. We compute the terms of order $|\rho|$ and $|\rho|^{0}$ in $X_{\rho}$. Since $a_{\rho}(x, 0)=a_{\rho}^{(0)}(x, 0)+O\left(|\rho|^{-1}\right)$, we may write

$$
\begin{aligned}
& P(\rho) a_{\rho}(x, 0) y_{0, \rho}+P(\rho) R_{\rho} y_{0, \rho}+P(\rho) a_{\rho}(x, 0) \psi_{\rho}+\left(P(\nabla) a_{\rho}(x, 0)\right) y_{0, \rho}+V^{\prime} a_{\rho}(x, 0) y_{0, \rho} \\
&=P(\rho) a_{\rho}^{(0)}(x, 0) y_{0, \rho}+P(\rho)\left(w_{1}, \vec{w}_{2}, \vec{w}_{3}, w_{4}\right)^{\prime}+P(\rho)\left(u_{1}, \vec{u}_{2}, \vec{u}_{3}, u_{4}\right)^{\prime} \\
&+P(\rho) a_{\rho}^{(0)}(x, 0)\left(\psi_{1}, \vec{\psi}_{2}, \vec{\psi}_{3}, \psi_{4}\right)^{\prime}+\left(P(\nabla) a_{\rho}^{(0)}(x, 0)\right) y_{0, \rho} \\
&+V^{\prime} a_{\rho}^{(0)}(x, 0) y_{0, \rho}+O\left(|\rho|^{-1}\right),
\end{aligned}
$$

where all of $w_{j}, u_{j}, \psi_{j}$ are $O\left(|\rho|^{-1}\right)$. Computing the $F_{1}$ and $F_{2}$ projections of this, we find that the fields are of the form

$$
\begin{aligned}
E=e^{x \cdot \rho} & {\left[\left(\vec{a}_{1 \cdot} \cdot y_{0, \rho}\right) \rho+\rho \wedge y_{567}+\left(w_{1}+u_{1}+\left(\vec{a}_{1} \cdot \psi_{\rho}\right)\right) \rho\right.} \\
& \left.+\rho \wedge\left(\vec{w}_{3}+\vec{u}_{3}+\vec{\psi}_{3}\right)+\nabla \vec{a}_{1} \cdot y_{0, \rho}-i \omega \mu y_{234}-i \omega \beta y_{567}+O\left(|\rho|^{-1}\right)\right]
\end{aligned}
$$

and

$$
\begin{aligned}
H=e^{x \cdot \rho} & {\left[\left(\vec{a}_{8 \cdot} \cdot y_{0, \rho}\right) \rho-\rho \wedge y_{234}+\left(w_{4}+u_{4}+\left(\vec{a}_{8} \cdot \psi_{\rho}\right)\right) \rho\right.} \\
& \left.-\rho \wedge\left(\vec{w}_{2}+\vec{u}_{2}+\vec{\psi}_{2}\right)+\nabla \vec{a}_{8 \cdot} \cdot y_{0, \rho}+i \omega \beta y_{234}-i \omega \varepsilon y_{567}+O\left(|\rho|^{-1}\right)\right] .
\end{aligned}
$$

We have used $y_{234}=\left(y_{2}, y_{3}, y_{4}\right)^{\prime}$ and $y_{567}=\left(y_{5}, y_{6}, y_{7}\right)^{\prime}$. We now make some choices for $\rho_{j}$. Fix $k \in \mathbb{R}^{3}$, and for $s \in \mathbb{R}, s>0$, let $\eta, \xi \in \mathbb{R}^{3}$ be such that

$$
\begin{gathered}
\langle\eta, k\rangle=\langle\eta, \xi\rangle=\langle k, \xi\rangle=0, \\
|\eta|^{2}=\frac{|k|^{2}}{4}+s^{2}+\omega^{2} \varepsilon_{0} \mu_{0}, \\
|\xi|^{2}=1 .
\end{gathered}
$$

Set

$$
\begin{aligned}
& \rho_{1}=\eta+i\left(\frac{k}{2}+s \xi\right), \\
& \rho_{2}=-\eta+i\left(\frac{k}{2}-s \xi\right),
\end{aligned}
$$

so that

$$
\begin{gathered}
\rho_{1}+\rho_{2}=i k, \\
\rho_{j} \cdot \rho_{j}=\omega^{2} \varepsilon_{0} \mu_{0}, \quad j=1,2 .
\end{gathered}
$$

Define $\tau_{j}=\lim _{s \rightarrow \infty} \rho_{j} / s$ and observe that $\tau_{1}=-\tau_{2}$. The parameter $s$ controls the growth of $|\rho|$; that is, $|\rho| \rightarrow \infty$ as $s \rightarrow \infty$. 
We must compute the highest order terms in the dot products of the fields to use in the identity (2.6). Each field is of order one, and so we might expect order two terms in the products; this fails to be the case for the following reason. If $y_{0, \rho_{1}}$ and $y_{0, \rho_{2}}$ are chosen to satisfy the condition of proposition 4.4 and $y_{1}=\left(y_{0, \rho_{1}}\right)_{234}, y_{2}=$ $\left(y_{0, \rho_{2}}\right)_{234}$ for example, then $y_{j} \cdot \rho_{j}=0$ and we find that

$$
\begin{aligned}
\rho_{1} \cdot \rho_{2} & =O\left(|\rho|^{0}\right), \\
\rho_{1} \cdot\left(y_{2} \wedge \rho_{2}\right) & =O\left(|\rho|^{0}\right), \\
\left(\rho_{1} \wedge y_{1}\right) \cdot\left(\rho_{2} \wedge y_{2}\right) & =\left(\rho_{1} \cdot \rho_{2}\right)\left(y_{1} \cdot y_{2}\right)-\left(\rho_{1} \cdot y_{2}\right)\left(\rho_{2} \cdot y_{1}\right) \\
& =\left(\rho_{1} \cdot \rho_{2}\right)\left(y_{1} \cdot y_{2}\right)-\left(\left(i k-\rho_{2}\right) \cdot y_{2}\right)\left(\left(i k-\rho_{1}\right) \cdot y_{1}\right) \\
& =O\left(|\rho|^{0}\right) .
\end{aligned}
$$

Thus the terms which appear to be of order two are in fact of order zero.

We must therefore compute the order one terms in the products of the fields. We shall choose $y_{0, \rho_{j}}$ of the form $y_{0, \rho_{j}}=\left(\delta_{1}, \overrightarrow{0}, \overrightarrow{0}, \delta_{2}\right)^{\prime}$ with $\delta_{j} \in\{0,1\}$. This simplifies the expressions (5.1) and (5.2) for $E$ and $H$. Choose first $y_{0, \rho_{1}}=y_{0, \rho_{2}}=(1, \overrightarrow{0}, \overrightarrow{0}, 0)^{\prime}$. Then

$$
\begin{aligned}
\lim _{s \rightarrow \infty} \frac{1}{s} E_{1} \cdot H_{2} & =e^{i x \cdot k}\left[a_{11}^{1} \tau_{1} \cdot \nabla a_{81}^{2}+a_{81}^{2} \tau_{2} \cdot \nabla a_{11}^{1}\right], \\
\lim _{s \rightarrow \infty} \frac{1}{s} E_{2} \cdot H_{1} & =e^{i x \cdot k}\left[a_{11}^{2} \tau_{1} \cdot \nabla a_{81}^{1}+a_{81}^{1} \tau_{2} \cdot \nabla a_{11}^{2}\right], \\
\lim _{s \rightarrow \infty} \frac{1}{s} E_{1} \cdot E_{2} & =e^{i x \cdot k}\left[a_{11}^{1} \tau_{1} \cdot \nabla a_{11}^{2}+a_{11}^{2} \tau_{2} \cdot \nabla a_{11}^{1}\right], \\
\lim _{s \rightarrow \infty} \frac{1}{s} H_{1} \cdot H_{2} & =e^{i x \cdot k}\left[a_{81}^{1} \tau_{1} \cdot \nabla a_{81}^{2}+a_{81}^{2} \tau_{2} \cdot \nabla a_{81}^{1}\right],
\end{aligned}
$$

where $a_{i j}^{l}$ is the $i j$ component of $a_{\rho_{l}}^{(0)}(x, 0)$. Now $\tau_{1}=-\tau_{2}$ and, by (7.2) from the proof of proposition 4.2 ,

$$
\tau_{2} \cdot \nabla a_{\rho_{2}}^{(0)}(x, 0)=-n_{\tau_{2}} a_{\rho_{2}}^{(0)}(x, 0),
$$

where $n_{\tau_{2}}=\lim _{s \rightarrow \infty} n_{\rho_{2}} / 2 s$. Thus

$$
\tau_{1} \cdot \nabla a_{i j}^{2}=\left(n_{\tau_{2}} a_{\rho_{2}}^{(0)}(x, 0)\right)_{i j} \text { and } \tau_{2} \cdot \nabla a_{i j}^{1}=\left(n_{\tau_{1}} a_{\rho_{1}}^{(0)}(x, 0)\right)_{i j}
$$

If $\vec{v}_{i j}^{l}$ denotes the $i j$ component of $V_{0}$ for parameters $\varepsilon_{l}, \mu_{l}, \beta_{l}$ (see section [3), and $\tau=\tau_{1}=-\tau_{2}$, then

$$
\begin{aligned}
\lim _{s \rightarrow \infty} \frac{1}{s} \int_{\Omega}\left(\left(\beta_{1}-\beta_{2}\right)\left(H_{1} \cdot E_{2}+H_{2} \cdot E_{1}\right)+\left(\varepsilon_{1}-\varepsilon_{2}\right) E_{1} \cdot E_{2}+\left(\mu_{2}-\mu_{1}\right) H_{1} \cdot H_{2}\right) d x \\
=\int_{\mathbb{R}^{3}} e^{i x \cdot k}\left\{( \beta _ { 1 } - \beta _ { 2 } ) \left[a_{81}^{2}\left(a_{11}^{1} \vec{v}_{12}^{1}+a_{81}^{1} \vec{v}_{13}^{1}\right)-a_{11}^{1}\left(a_{11}^{2} \vec{v}_{42}^{2}+a_{81}^{2} \vec{v}_{43}^{2}\right)\right.\right. \\
\left.\quad+a_{11}^{2}\left(a_{11}^{1} \vec{v}_{42}^{1}+a_{81}^{1} \vec{v}_{43}^{1}\right)-a_{81}^{1}\left(a_{11}^{2} \vec{v}_{12}^{2}+a_{81}^{2} \vec{v}_{13}^{2}\right)\right] \cdot \tau \\
\quad+\left(\varepsilon_{1}-\varepsilon_{2}\right)\left[a_{11}^{2}\left(a_{11}^{1} \vec{v}_{12}^{1}+a_{81}^{1} \vec{v}_{13}^{1}\right)-a_{11}^{1}\left(a_{11}^{2} \vec{v}_{12}^{2}+a_{81}^{2} \vec{v}_{13}^{2}\right)\right] \cdot \tau \\
\left.\quad+\left(\mu_{2}-\mu_{1}\right)\left[a_{81}^{2}\left(a_{11}^{1} \vec{v}_{42}^{1}+a_{81}^{1} \vec{v}_{43}^{1}\right)-a_{81}^{1}\left(a_{11}^{2} \vec{v}_{42}^{2}+a_{81}^{2} \vec{v}_{43}^{2}\right)\right] \cdot \tau\right\} d x=0 .
\end{aligned}
$$

The integration extends to all of $\mathbb{R}^{3}$ since the parameters have been extended to agree outside $\Omega$. This identity holds for any $k \in \mathbb{R}^{3}$, and so the Fourier transform of the integrand, and hence the integrand itself, is zero. Repeating these calculations 
for the choices

$$
\begin{array}{ll}
y_{0, \rho_{1}}=(1, \overrightarrow{0}, \overrightarrow{0}, 0), & y_{0, \rho_{2}}=(0, \overrightarrow{0}, \overrightarrow{0}, 1) \\
y_{0, \rho_{1}}=(0, \overrightarrow{0}, \overrightarrow{0}, 1), & y_{0, \rho_{2}}=(1, \overrightarrow{0}, \overrightarrow{0}, 0) \\
y_{0, \rho_{1}}=(0, \overrightarrow{0}, \overrightarrow{0}, 1), & y_{0, \rho_{2}}=(0, \overrightarrow{0}, \overrightarrow{0}, 1)
\end{array}
$$

and rearranging, we obtain the identity

$$
\begin{aligned}
\left(\begin{array}{cc}
a_{81}^{1} & a_{11}^{1} \\
a_{88}^{1} & a_{18}^{1}
\end{array}\right)\left\{\left(\begin{array}{cc}
\vec{v}_{22}^{1}-\vec{v}_{11}^{2} & \vec{v}_{12}^{1}-\vec{v}_{12}^{2} \\
\vec{v}_{21}^{1}-\vec{v}_{21}^{2} & \vec{v}_{11}^{1}-\vec{v}_{22}^{2}
\end{array}\right) \cdot \tau\left(\beta_{1}-\beta_{2}\right)\right. \\
+\left(\begin{array}{cc}
\vec{v}_{12}^{1} & 0 \\
\vec{v}_{11}^{1}-\vec{v}_{11}^{2} & -\vec{v}_{12}^{2}
\end{array}\right) \cdot \tau\left(\varepsilon_{1}-\varepsilon_{2}\right) \\
\\
\left.\quad+\left(\begin{array}{cc}
-\vec{v}_{12}^{2} & \vec{v}_{22}^{1}-\vec{v}_{22}^{2} \\
0 & \vec{v}_{21}^{2}
\end{array}\right) \cdot \tau\left(\mu_{2}-\mu_{1}\right)\right\}\left(\begin{array}{ll}
a_{11}^{2} & a_{18}^{2} \\
a_{81}^{2} & a_{88}^{2}
\end{array}\right)=0 .
\end{aligned}
$$

The two matrices involving the $a_{i j}^{l}$ are invertible by construction, and may be removed from the identity. If any one of the pairs of parameters is equal, then this system implies that the other two are equal throughout $\Omega$. We illustrate this in the practically most applicable case, when $\mu_{1}=\mu_{2}$. Assume now that $\mu_{1}=\mu_{2}=\mu$; we obtain the following four equations. Let $D_{j}=\varepsilon_{j} \mu+\beta_{j}^{2}$, and for simplicity of exposition, let us use $\nabla_{\tau}$ for $\nabla \cdot \tau$. Then

$$
\begin{aligned}
\left(D_{1}\left(\mu \nabla_{\tau} \varepsilon_{2}+\beta_{2} \nabla_{\tau} \beta_{2}\right)-D_{2}\left(\varepsilon_{1} \nabla_{\tau} \mu+\beta_{1} \nabla_{\tau} \beta_{1}\right)\right)\left(\beta_{1}-\beta_{2}\right) & \\
- & D_{2}\left(\mu \nabla_{\tau} \beta_{1}-\beta_{1} \nabla_{\tau} \mu\right)\left(\varepsilon_{1}-\varepsilon_{2}\right)=0, \\
\left(D_{1}\left(\mu \nabla_{\tau} \beta_{2}-\beta_{2} \nabla_{\tau} \mu\right)-D_{2}\left(\mu \nabla_{\tau} \beta_{1}-\beta_{1} \nabla_{\tau} \mu\right)\right)\left(\beta_{1}-\beta_{2}\right)=0, & \\
\left(D_{1}\left(\beta_{2} \nabla_{\tau} \varepsilon_{2}-\varepsilon_{2} \nabla_{\tau} \beta_{2}\right)-D_{2}\left(\beta_{1} \nabla_{\tau} \varepsilon_{1}-\varepsilon_{1} \nabla_{\tau} \beta_{1}\right)\right)\left(\beta_{1}-\beta_{2}\right) & \\
+\left(D_{1}\left(\mu \nabla_{\tau} \varepsilon_{2}+\beta_{2} \nabla_{\tau} \beta_{2}\right)-D_{2}\left(\mu \nabla_{\tau} \varepsilon_{1}+\beta_{1} \nabla_{\tau} \beta_{1}\right)\right)\left(\varepsilon_{1}-\varepsilon_{2}\right) & =0, \\
\left(D_{1}\left(\varepsilon_{2} \nabla_{\tau} \mu+\beta_{2} \nabla_{\tau} \beta_{2}\right)-D_{2}\left(\mu \nabla_{\tau} \varepsilon_{1}+\beta_{1} \nabla_{\tau} \beta_{1}\right)\right)\left(\beta_{1}-\beta_{2}\right) & \\
+D_{1}\left(\mu \nabla_{\tau} \beta_{2}-\beta_{2} \nabla_{\tau} \mu\right)\left(\varepsilon_{1}-\varepsilon_{2}\right) & =0 .
\end{aligned}
$$

Lemma 5.4. For all $x \in \mathbb{R}^{3}, \nabla_{\tau} \log \left(D_{1} / D_{2}\right)=0$.

Proof. Case I. Assume that $\beta_{1}(x)-\beta_{2}(x)=0$ (we shall suppress the explicit evaluation at $x$ ). If also $\varepsilon_{1}-\varepsilon_{2}=0$ then we are done; otherwise, (5.4) and (5.7) imply $\mu \nabla_{\tau} \beta=\beta \nabla_{\tau} \mu$. Now since $\nabla_{\tau} D_{j}=\varepsilon_{j} \nabla_{\tau} \mu+\mu \nabla_{\tau} \varepsilon_{j}+2 \beta_{j} \nabla_{\tau} \beta_{j}$, (5.6) gives

$$
D_{1}\left(\nabla_{\tau} D_{2}-\varepsilon_{2} \nabla_{\tau} \mu-\beta \nabla_{\tau} \beta\right)-D_{2}\left(\nabla_{\tau} D_{1}-\varepsilon_{1} \nabla_{\tau} \mu-\beta \nabla_{\tau} \beta\right)=0,
$$

and expanding the $D_{1}$ and $D_{2}$ in this we obtain

$$
\begin{aligned}
D_{1} \nabla_{\tau} D_{2}-D_{2} \nabla_{\tau} D_{1} & =\left(\varepsilon_{1}-\varepsilon_{2}\right) \beta\left(\beta \nabla_{\tau} \mu-\mu \nabla_{\tau} \beta\right) \\
& =0 .
\end{aligned}
$$

Thus $\nabla_{\tau} \log \left(D_{1} / D_{2}\right)=D_{1} \nabla_{\tau} D_{2}-D_{2} \nabla_{\tau} D_{1}=0$.

Case II. If $\beta_{1}-\beta_{2} \neq 0$, then (5.4) $+(5.7$ ) gives

$$
\begin{aligned}
\left(D_{1} \nabla_{\tau} D_{2}\right. & \left.-D_{2} \nabla_{\tau} D_{1}\right)\left(\beta_{1}-\beta_{2}\right) \\
& =\left(D_{2}\left(\mu \nabla_{\tau} \beta_{1}-\beta_{1} \nabla_{\tau} \mu\right)-D_{1}\left(\mu \nabla_{\tau} \beta_{2}-\beta_{2} \nabla_{\tau} \mu\right)\right)\left(\varepsilon_{1}-\varepsilon_{2}\right)=0
\end{aligned}
$$

by (5.5). Thus again $\nabla_{\tau} \log \left(D_{1} / D_{2}\right)=D_{1} \nabla_{\tau} D_{2}-D_{2} \nabla_{\tau} D_{1}=0$. 
Finally, we must show that lemma 5.4 finishes the proof of theorem 2.2. Since $\nabla_{\tau} \log \left(D_{1} / D_{2}\right)=0$ in $\mathbb{R}^{3}, D_{1} / D_{2}$ is constant, and hence $D_{1}=D_{2}$ since this is true outside $\Omega$. Now (5.4) (5.7) together with $D_{1}=D_{2}$ implies

$$
\left(\mu \nabla_{\tau} \beta_{1}-\beta_{1} \nabla_{\tau} \mu-\mu \nabla_{\tau} \beta_{2}+\beta_{2} \nabla_{\tau} \mu\right)\left(\varepsilon_{1}-\varepsilon_{2}\right)=0 ;
$$

if $\mu \nabla_{\tau} \beta_{1}-\beta_{1} \nabla_{\tau} \mu=\mu \nabla_{\tau} \beta_{2}-\beta_{2} \nabla_{\tau} \mu$, then

$$
\mu^{2} \nabla_{\tau} \frac{\beta_{1}}{\mu}=\mu^{2} \nabla_{\tau} \frac{\beta_{2}}{\mu}
$$

and so $\left(\beta_{1}-\beta_{2}\right) / \mu$ is constant. This constant is zero since $\beta_{1}=\beta_{2}$ outside $\Omega$, and hence $\beta_{1}=\beta_{2}$ everywhere. Then (5.6) implies that $\mu \nabla_{\tau}\left(\varepsilon_{1}-\varepsilon_{2}\right)=0$, and so, similarly, $\varepsilon_{1}=\varepsilon_{2}$. On the other hand, if in (5.8) $\varepsilon_{1}=\varepsilon_{2}$, then by (5.6) $\varepsilon \nabla_{\tau} \beta_{1}-\beta_{1} \nabla_{\tau} \varepsilon=\varepsilon \nabla_{\tau} \beta_{2}-\beta_{2} \nabla_{\tau} \varepsilon$ and, in the same manner as above, $\left(\beta_{1}-\beta_{2}\right) / \varepsilon$ is constant and again $\beta_{1}=\beta_{2}$.

\section{Appendix A}

Proof of Theorem 2.1. We define the function spaces

$$
\begin{aligned}
& H(\nabla \wedge)=\left\{E \in L^{2}(\Omega)^{3} \mid \nabla \wedge E \in L^{2}(\Omega)^{3}\right\} \\
& \stackrel{\circ}{H}(\nabla \wedge)=\left\{E \in H(\nabla \wedge) \mid \int_{\Omega} \nabla \wedge E \cdot F=\int_{\Omega} E \cdot \nabla \wedge F \text { for all } F \in H(\nabla \wedge)\right\} .
\end{aligned}
$$

We shall use the equivalent Born-Fedorov formulation

$$
\begin{aligned}
\nabla \wedge E & =i \omega \tilde{\mu} H+i \omega \tilde{\mu} \tilde{\beta} \nabla \wedge H, \\
\nabla \wedge H & =-i \omega \tilde{\varepsilon} E-i \omega \tilde{\varepsilon} \tilde{\beta} \nabla \wedge E,
\end{aligned}
$$

which, following the presentation of [11, we may write as

$$
(L-\omega-B)\left(\begin{array}{c}
E \\
H
\end{array}\right)=0
$$

where

$$
\begin{aligned}
L & =\left[\begin{array}{cc}
-i \nabla \wedge & 0 \\
0 & -i \nabla \wedge
\end{array}\right]: \mathcal{D}(L) \rightarrow L^{2}(\Omega)^{3} \times L^{2}(\Omega)^{3} \\
B & =\frac{\omega}{\omega^{2} \tilde{\varepsilon} \tilde{\mu} \tilde{\beta}^{2}-1}\left[\begin{array}{cc}
1 & -\tilde{\mu} \\
\tilde{\varepsilon} & 1
\end{array}\right] .
\end{aligned}
$$

The domain of $L$ is $\mathcal{D}(L)=\stackrel{\circ}{H}(\nabla \wedge) \times H(\nabla \wedge)$; on $\mathcal{D}(L), L$ is self-adjoint. In order to solve Maxwell's equations with

$$
\left.\nu \wedge E\right|_{\partial \Omega}=F \in T H_{\text {Div }}^{\frac{1}{2}}(\partial \Omega)
$$

we write $\tilde{E}=E-R F$, where $R$ is the right inverse of the tangential trace mapping

$$
t r: H^{1}(\Omega)^{3} \rightarrow T H_{\text {Div }}^{\frac{1}{2}}(\partial \Omega), \quad t r:\left.E \mapsto \nu \wedge E\right|_{\partial \Omega} .
$$

Then the boundary value problem may be written

$$
(L-\omega-B)\left(\begin{array}{c}
\tilde{E} \\
H
\end{array}\right)=\left(\begin{array}{c}
J \\
K
\end{array}\right)
$$

where

$$
J=i \nabla \wedge R F+\frac{\omega^{3} \tilde{\varepsilon} \tilde{\mu} \tilde{\beta}^{2}}{\omega^{2} \tilde{\varepsilon} \tilde{\mu} \tilde{\beta}^{2}-1} R F, \quad K=\frac{\omega \tilde{\varepsilon}}{\omega^{2} \tilde{\varepsilon} \tilde{\mu} \tilde{\beta}^{2}-1} R F
$$


Lemma 6.1. The range of $L, \mathcal{R}(L)$, is closed, the mapping $L^{-1}: \mathcal{R}(L) \rightarrow \mathcal{R}(L) \cap$ $\mathcal{D}(L)$ exists, and $L^{-1}$ is continuous and compact.

This is proven in 3 . From this we have

$$
L^{2}(\Omega)^{3} \times L^{2}(\Omega)^{3}=\operatorname{Ker}(L) \oplus \mathcal{R}(L),
$$

and there is a discrete set $\mathcal{S} \subset \mathbb{R}$ containing no limit points such that $(L-\omega)^{-1}$ exists and is compact for all $\omega \in \mathbb{C} \backslash \mathcal{S}$. The compactness follows from

$$
(L-w)^{-1}=L^{-1}+\omega L^{-1}(L-w)^{-1}
$$

and the compactness of $L^{-1}$. For $\omega \notin \mathcal{S}$, we want to solve

$$
\left(I-(L-\omega)^{-1} B\right)\left(\begin{array}{c}
\tilde{E} \\
H
\end{array}\right)=(L-\omega)^{-1}\left(\begin{array}{c}
J \\
K
\end{array}\right) .
$$

Recall that $\omega^{2} \tilde{\varepsilon} \tilde{\mu} \tilde{\beta}^{2}-1 \neq 0$ for $\omega \in \mathcal{A}=\mathbb{C} \backslash\left\{\omega|\omega \in \mathbb{R},| \omega \mid \geq \omega_{0}\right\}$; so on $\mathcal{A} \backslash \mathcal{S}, B$ is analytic and $(L-\omega)^{-1}$ exists; at $\omega=0$ we have $B=0$, so by the analytic Fredholm theorem (for example 9]), $\left(I-(L-\omega)^{-1} B\right)^{-1}$ exists for all $\omega \in \mathcal{A} \backslash\left(\mathcal{S} \cup \mathcal{S}^{\prime}\right)$ for some discrete set $\mathcal{S}^{\prime}$ containing no limit points in $\mathcal{A} \backslash \mathcal{S}$. The theorem follows with $D=\mathcal{S} \cup \mathcal{S}^{\prime}$

\section{Appendix B}

Proof of Proposition 4.2. Let $S_{\rho}=\operatorname{Char}\left(\Delta_{\rho}\right)=\left\{\left.\xi \in \mathbb{R}^{3}|-| \xi\right|^{2}+2 i \rho \cdot \xi-\omega^{2} \varepsilon_{0} \mu_{0}=\right.$ $0\}$. In a neighborhood of $S_{\rho}$, we will construct $A_{\rho}=B_{\rho}$, and so in such a neighborhood, (4.2) is equivalent to

$$
\left[\Delta_{\rho}, A_{\rho}\right]+N_{\rho}^{+} A_{\rho}-A_{\rho} \varphi C_{\rho} \varphi=0 .
$$

We define $A_{\rho}$ by defining its symbol $a_{\rho}(x, \xi) \in S^{0}\left(\mathbb{R}^{3} \times \mathbb{R}^{3} \times Z\right)^{8 \times 8}$, an $8 \times 8$ matrix. Write

$$
\rho=\eta+i k, \quad \text { with } \quad \eta, k \in \mathbb{R}^{3} .
$$

Computing terms of homogeneity of order 1 in $\xi$ and $\rho$ in (7.1), we have

$$
\left(L_{1}+i L_{2}\right) a_{\rho}^{(0)}+\frac{1}{2|\rho|} n_{\rho} a_{\rho}^{(0)}=0,
$$

where $a_{\rho}^{(0)}$ is the principal symbol of $A_{\rho}$,

$$
L_{1}=\frac{1}{|\rho|} \sum_{j=1}^{3} \eta_{j} \frac{\partial}{\partial x_{j}}, \quad L_{2}=\frac{1}{|\rho|} \sum_{j=1}^{3}\left(k_{j}+\xi_{j}\right) \frac{\partial}{\partial x_{j}},
$$

and $n_{\rho}$ is the principal symbol of $N_{\rho}^{+}$. Observe that so long as $L_{1}$ and $L_{2}$ are linearly independent, there is a change of variables mapping $L_{1}+i L_{2}$ to $\bar{\partial}$ where

$$
\bar{\partial}=\frac{1}{2}\left(\frac{\partial}{\partial x_{1}}+i \frac{\partial}{\partial x_{2}}\right) ;
$$

in some of the proofs that follow we shall assume that $L_{1}+i L_{2}=\bar{\partial}$ to simplify the exposition. It is easy to see that $L_{1}$ and $L_{2}$ are linearly independent on and hence near $S_{\rho}$, which for fixed $\rho=\eta+i k$ is the circle orthogonal to $\eta$ of radius $|k|-\omega^{2} \varepsilon_{0} \mu_{0}$ and center $-k$ (we take $|\rho|$ sufficiently large so that $|k|-\omega^{2} \varepsilon_{0} \mu_{0}>0$ ). 
We now describe a partition of unity of $\mathbb{R}_{\xi}^{3}$-space which depends smoothly on $\rho$, and which divides the space into a tubular neighborhood of $S_{\rho}$ and the complement. Let

$$
\begin{array}{ll}
\mathcal{U}_{1, \rho}=\left\{\xi \in \mathbb{R}^{3}|| \xi-S_{\rho}\left|<\frac{2}{3 \sqrt{2}}\right| \rho \mid\right\}, & \mathcal{U}_{1, \rho}^{0}=\left\{\xi \in \mathbb{R}^{3}|| \xi-S_{\rho}\left|<\frac{1}{3 \sqrt{2}}\right| \rho \mid\right\}, \\
\mathcal{U}_{2, \rho}=\left\{\xi \in \mathbb{R}^{3}|| \xi-S_{\rho}\left|>\frac{1}{3 \sqrt{2}}\right| \rho \mid\right\}, & \mathcal{U}_{2, \rho}^{0}=\left\{\xi \in \mathbb{R}^{3}|| \xi-S_{\rho}\left|>\frac{2}{3 \sqrt{2}}\right| \rho \mid\right\} .
\end{array}
$$

For $|\rho|=1$ let $\left\{\tilde{\varphi}_{1, \rho}, \tilde{\varphi}_{2, \rho}\right\}$ be a partition of unity subordinate to the open cover $\left\{\mathcal{U}_{1, \rho}, \mathcal{U}_{2, \rho}\right\}$ of $\mathbb{R}_{\xi}^{3}$, depending smoothly on $\rho$, and such that

$$
\tilde{\varphi}_{1, \rho}=1 \text { on } \mathcal{U}_{1, \rho}^{0} \quad \text { and } \quad \tilde{\varphi}_{2, \rho}=0 \text { on } \mathcal{U}_{1, \rho}^{0} .
$$

Then $\left\{\xi \mid \tilde{\varphi}_{1, \rho}(\xi)=1\right.$ and $\left.\tilde{\varphi}_{2, \rho}(\xi)=0\right\}$ is a tubular neighborhood of $S_{\rho}$ of radius $|\rho| / 3 \sqrt{2}$. On this neighborhood, $L_{1}$ and $L_{2}$ are linearly independent. Now extend $\tilde{\varphi}_{j, \rho}$ to all of $\mathbb{R}_{\xi}^{3} \times Z$ to be homogeneous of degree zero in $(\xi, \rho)$ for $|\rho|>1$ say, and arbitrarily for $|\rho|<1$; that is, define

$$
\tilde{\varphi}_{j, \rho}(\xi)=\tilde{\varphi}_{j, \frac{\rho}{|\rho|}}\left(\frac{\xi}{|\rho|}\right),
$$

so

$$
\tilde{\varphi}_{j, \lambda \rho}(\lambda \xi)=\tilde{\varphi}_{j, \frac{\lambda \rho}{\lambda|\rho|}}\left(\frac{\lambda \xi}{\lambda|\rho|}\right)=\tilde{\varphi}_{j, \frac{\rho}{|\rho|}}\left(\frac{\xi}{|\rho|}\right)=\tilde{\varphi}_{j, \rho}(\xi) .
$$

Proposition 7.1. Let $-1<\delta<0$. There is a unique $a_{\rho}^{(0)}(x, \xi) \in S^{0}\left(\mathbb{R}^{3}, Z\right)$ solving (7.2) with $a_{\rho}^{(0)}-I \in L_{\delta}^{2}\left(\mathbb{R}_{x}^{3}\right)$; furthermore, $a_{\rho}^{(0)}$ is invertible for large $\rho$.

Proof. We shall only need the solution on the support of $\tilde{\varphi}_{1, \rho}$ where $L_{1}$ and $L_{2}$ are linearly independent, and so we shall prove the result for $\bar{\partial}$ :

$$
\bar{\partial} a_{\rho}^{(0)}+\frac{1}{2|\rho|} n_{\rho} a_{\rho}^{(0)}=0 .
$$

Write $a_{\rho}^{(0)}=d_{\rho}+I$, and $\tilde{d}_{\rho}=\bar{\partial} d_{\rho}$; thus we must solve

$$
\left(I+\frac{1}{2|\rho|} n_{\rho} \bar{\partial}^{-1}\right) \tilde{d}_{\rho}=-\frac{1}{2|\rho|} n_{\rho} .
$$

We shall need the following lemmas.

Lemma 7.2. If $-1<\delta<0$, then

$$
\frac{1}{2|\rho|} n_{\rho} \bar{\partial}^{-1}: L_{\delta+1}^{2}\left(\mathbb{R}_{x}^{3}\right) \rightarrow L_{\delta+1}^{2}\left(\mathbb{R}_{x}^{3}\right)
$$

is compact.

Proof. From [6], Theorem 2.1 (with $n=3, p=p^{\prime}=2, \rho=\delta, m=1, r=0$ ), for $v \in C_{0}^{\infty}\left(\mathbb{R}^{3}\right)$ we have

$$
\|v\|_{H_{\delta}^{1}} \leq C\|\bar{\partial} v\|_{L_{\delta+1}^{2}},
$$

and since $H_{\delta}^{1}$ is the completion of $C_{0}^{\infty}\left(\mathbb{R}^{3}\right)$ in this norm, the same estimate holds for all $v \in H_{\delta}^{1}\left(\mathbb{R}^{3}\right)$ such that $\bar{\partial} v \in L_{\delta+1}^{2}$. Thus

$$
\bar{\partial}^{-1}: L_{\delta+1}^{2} \rightarrow H_{\delta}^{1}
$$


continuously. Since $n_{\rho}$ is compactly supported (in $x$ ), we have

$$
L_{\delta+1}^{2} \underset{c t s}{\stackrel{\bar{\partial}^{-1}}{\longrightarrow}} H_{\delta}^{1} \underset{c t s}{\stackrel{n_{\rho}}{c t}} H^{1}\left(\operatorname{supp}\left(n_{\rho}\right)\right) \underset{\text { compact }}{\stackrel{i n c l}{\longrightarrow}} L^{2}\left(\operatorname{supp}\left(n_{\rho}\right)\right) \underset{c t s}{\stackrel{i n c l}{\longrightarrow}} L_{\delta+1}^{2}
$$

Lemma 7.3. The equation

$$
\left(I+\frac{1}{2|\rho|} n_{\rho} \bar{\partial}^{-1}\right) \tilde{d}_{\rho}=0
$$

has only the trivial solution in $L_{\delta+1}^{2}\left(\mathbb{R}_{x}^{3}\right)$.

Proof. With $d_{\rho}=\bar{\partial}^{-1} \tilde{d}_{\rho}$, we show that $d_{\rho}=0$ is the unique solution in $L_{\delta}^{2}$ to

$$
\bar{\partial} d_{\rho}+\frac{1}{2|\rho|} n_{\rho} d_{\rho}=0 .
$$

Since $\operatorname{supp}\left(n_{\rho}\right) \subset\{z|| z \mid \leq R\}$ for some $R, d_{\rho}$ is analytic for $|z|>R$. From

$$
d_{\rho}(z)=\frac{-1}{2 \pi i} \int_{|z| \leq R} \frac{1}{z-w} \frac{n_{\rho}(w) d_{\rho}(w)}{2|\rho|} d w \wedge d \bar{w}
$$

it follows easily that $d_{\rho}(z)$ decays to all orders at infinity; since $d_{\rho}$ is also analytic in a neighborhood of infinity, it follows that $d_{\rho}(z)=0$ in a neighborhood of infinity. Now by (Cor. 5.3.8, [14]) unique continuation implies $d_{\rho}(z)$ is identically zero.

From the above lemmas and the Fredholm alternative, there is a unique $\tilde{d}_{\rho} \in$ $L_{\delta+1}^{2}$ soving (7.4); or, if we write $a_{\rho}^{(0)}=I+\bar{\partial}^{-1} \tilde{d}_{\rho}$, then $a_{\rho}^{(0)}-I \in L_{\delta}^{2}\left(\mathbb{R}_{x}^{3}\right)$ and $a_{\rho}^{(0)}$ solves (7.3).

To prove that $a_{\rho}^{(0)}$ is invertible we exploit the structure of $n_{\rho}$ (see section 3 ):

$$
n_{\rho}=\left[\begin{array}{cccc}
\vec{v}_{12} \cdot(\rho+i \xi) & -\vec{v}_{13} \wedge(\rho+i \xi) & \vec{v}_{12} \wedge(\rho+i \xi) & \vec{v}_{13} \cdot(\rho+i \xi) \\
0 & 0 & 0 & 0 \\
0 & 0 & 0 & 0 \\
\vec{v}_{42} \cdot(\rho+i \xi) & -\vec{v}_{43} \wedge(\rho+i \xi) & \vec{v}_{42} \wedge(\rho+i \xi) & \vec{v}_{43} \cdot(\rho+i \xi)
\end{array}\right]
$$

This implies that $a_{\rho}^{(0)}$ is of the form

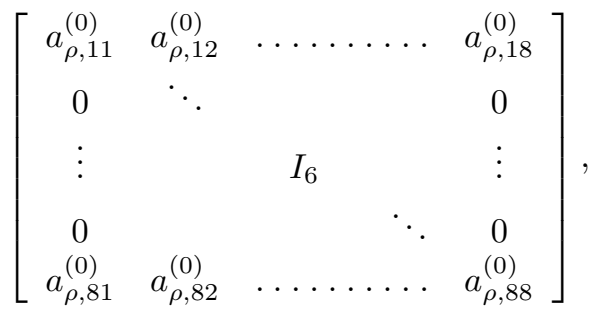

where $I_{6}$ is the $6 \times 6$ identity matrix. It follows that

$$
\operatorname{det}\left(a_{\rho}^{(0)}\right)=\operatorname{det}\left[\begin{array}{cc}
a_{\rho, 11}^{(0)} & a_{\rho, 18}^{(0)} \\
a_{\rho, 81}^{(0)} & a_{\rho, 88}^{(0)}
\end{array}\right]=\operatorname{det}\left(\tilde{a}_{\rho}^{(0)}\right), \text { say, }
$$

and $\tilde{a}_{\rho}^{(0)}$ satisfies

$$
\bar{\partial} \tilde{a}_{\rho}^{(0)}+\frac{1}{2|\rho|}\left[\begin{array}{cc}
n_{\rho, 11} & n_{\rho, 18} \\
n_{\rho, 81} & n_{\rho, 88}
\end{array}\right] \tilde{a}_{\rho}^{(0)}=0
$$


and so $\operatorname{det}\left(\tilde{a}_{\rho}^{(0)}\right)$ satisfies

$$
\bar{\partial} \operatorname{det}\left(\tilde{a}_{\rho}^{(0)}\right)+\operatorname{tr}\left[\begin{array}{ll}
n_{\rho, 11} & n_{\rho, 18} \\
n_{\rho, 81} & n_{\rho, 88}
\end{array}\right] \operatorname{det}\left(\tilde{a}_{\rho}^{(0)}\right)=0 .
$$

Furthermore, $a_{\rho}^{(0)} \in L_{\delta}^{2}\left(\mathbb{R}_{x}^{3}\right)$ implies $\left|\operatorname{det} a_{\rho}^{(0)}-1\right| \rightarrow 0$ as $|x| \rightarrow \infty$. By this and the compact support of $n_{\rho},(7.5)$ has a unique solution with $\operatorname{det}\left(\tilde{a}_{\rho}^{(0)}\right)-1 \in L_{\delta}^{2}(\mathbb{R})$ given by $\operatorname{det}\left(\tilde{a}_{\rho}^{(0)}\right)=e^{-\tau}$, where $\bar{\partial} \tau=(1 / 2|\rho|)\left(n_{\rho, 11}+n_{\rho, 88}\right)$. Thus $\operatorname{det} a_{\rho}^{(0)}=\operatorname{det} \tilde{a}_{\rho}^{(0)} \neq 0$, and $a_{\rho}^{(0)}$ is invertible. The smoothness of $a_{\rho}^{(0)}$ follows from differentiating equation (7.3) and from the fact that the change of coordinates transforming $L_{1}+i L_{2}$ to $\bar{\partial}$ is smooth.

We define $a_{\rho}^{(j)}$ for $j<0$ iteratively to be homoegeneous of order $j$ in $\xi$, and $\rho$ by considering terms of homogeneity $j+1$ in (7.1), and write $a_{\rho}$ as an asymptotic sum of the $a_{\rho}^{(j)}$. This completes the proof of proposition 7.1

Recall that we have been restricting ourselves to a neighborhood of $S_{\rho}$ where we may consider $L_{1}+i L_{2}$ to be $\bar{\partial}$; now define $a_{\rho}$ on all of $\mathbb{R}_{x}^{3} \times \mathbb{R}_{\xi}^{3} \times Z_{\rho}$ by taking $\tilde{\varphi}_{1, \rho} a_{\rho}+\tilde{\varphi}_{2, \rho} I$. Abusing notation, we shall call this $a_{\rho}$. Since $\tilde{\varphi}_{j, \rho}$ are homogeneous of degree 0 in $\xi$ and $\rho$, it follows that $a_{\rho} \in S^{0}\left(\mathbb{R}_{x}^{3} \times \mathbb{R}_{\xi}^{3} \times Z_{\rho}\right)$.

To achieve (4.2) we now define $C_{\rho} \in L^{0}\left(\mathbb{R}^{3}, Z\right)$ by

$$
A_{\rho} \varphi C_{\rho} \varphi=\left[\Delta_{\rho}, A_{\rho}\right]+N_{\rho}^{+} A_{\rho}
$$

for large $|\rho|$, so that $A_{\rho}$ is invertible. Next we define $B_{\rho} \in L^{0}\left(\mathbb{R}^{3}, Z\right)$ by

$$
B_{\rho}=\tilde{\varphi}_{1, \rho} A_{\rho}+\tilde{\varphi}_{2, \rho}\left(\Delta_{\rho}+N_{\rho}^{+}\right) A_{\rho}\left(\Delta_{\rho}+\varphi C_{\rho} \varphi\right)^{-1},
$$

observing that $\Delta_{\rho}+\varphi C_{\rho} \varphi$ is invertible on $\operatorname{supp} \tilde{\varphi}_{2, \rho}$, which is disjoint from $S_{\rho}$. To summarize, where $\tilde{\varphi}_{1, \rho}=1, A_{\rho}=B_{\rho}$ and we have (4.2) via (7.1); where $\tilde{\varphi}_{2, \rho}=1$, (7.7) gives (4.2), and in between,

$$
\begin{aligned}
B_{\rho}\left(\Delta_{\rho}+\varphi C_{\rho} \varphi\right) & =\tilde{\varphi}_{1, \rho} A_{\rho}\left(\Delta_{\rho}+\varphi C_{\rho} \varphi\right)+\tilde{\varphi}_{2, \rho}\left(\Delta_{\rho}+N_{\rho}^{+}\right) A_{\rho} \\
& =\left(\tilde{\varphi}_{1, \rho}+\tilde{\varphi}_{2, \rho}\right)\left(\Delta_{\rho}+N_{\rho}^{+}\right) A_{\rho}
\end{aligned}
$$

by (7.6). This completes the proof of proposition 4.2

\section{REFERENCES}

[1] Lee J. and Uhlmann G., 1989, Determining Anisotropic Real-Analytic Conductivities by Boundary Measurements Comm. Pure Appl. Math. 42 1097-1112 MR 91a:35166

[2] Lakhtakia A., Varadan V. K. and Varadan V. V., 1989, Time-Harmonic Electromagnetic Fields in Chiral Media, Lecture Notes in Physics, Vol 335 (Berlin: Springer-Verlag) MR 90e:78001

[3] Leis L., 1986, Initial Bounday Value Problems in Mathematical Physics (New York: John Wiley and Sons Inc.) MR 87h:35083

[4] McDowall S., 1997, Boundary determination of material parameters from electromagnetic boundary information Inverse Problems 13 153-163 MR 98c:78010

[5] Nakamura G. and Uhlmann G., 1994, Global uniqueness for an inverse boundary problem arising in elasticity Invent. math. 118 457-474 MR 95i:35313

[6] Nirenberg L. and Walker H.F., 1973, The Null Spaces of Elliptic Partial Differnetial Operators in $\mathbb{R}^{n}$ J. Math. Anal. Appl 42 271-301 MR 47:9354

[7] Ola P., Päivärinta L. and Somersalo E., 1993, An Inverse Boundary Problem in Electrodynamics Duke Math. J. 70 617-653 MR 94i:35196

[8] Ola P. and Somersalo E., 1996, Electromagnetic Inverse Problems and Generalized Sommerfeld Potentials SIAM J. Appl. Math. 56 1129-1145 MR 97b:35194

[9] Reed M. and Simon B., 1972, Methods of Modern Mathematical Physics, Vol. I (New York: Academic Press) MR 58:12429a 
[10] Shubin M. A., 1987, Pseudodifferential Operators and Spectral Theory (Berlin Heidelberg New York: Springer Series in Soviet Mathematics) MR 88c:47105

[11] Somersalo E., Isaacson D. and Cheney M., 1992, A Linearized Inverse Boundary Value Problem for Maxwell's Equations J. Comput. Appl. Math. 42 123-136 MR 93f:35242

[12] Sylvester J. and Uhlmann G., 1987, A global uniqueness theorem for an inverse boundary problem Annals of Math. 125 153-169 MR 88b:35205

[13] Tolmasky C., 1998, Exponentially growing solutions for non-smooth first-order perturbations of the Laplacian SIAM J. Math. Anal. 29 116-133

[14] Wendland W. L., 1979, Elliptic Systems in the Plane (London: Pitman) MR 80h:35053

Department of Mathematics, Universtiy of Washington, Box 354350, Seattle, WashINGTON 98195-4350

Current address: Department of Mathematics, University of Rochester, Rochester, New York 14627

E-mail address: mcdowall@math.rochester.edu 